\title{
Non-Relativistic Propagators via Schwinger's Method
}

\author{
A. Aragão, H. Boschi-Filho, C. Farina, \\ Instituto de Física, Universidade Federal do Rio de Janeiro, \\ Caixa Postal 68.528, 21941-972 Rio de Janeiro, RJ, Brazil. \\ and F. A. Barone \\ Universidade Federal de Itajubá, Av. BPS 1303 \\ Caixa Postal 50 - 37500-903, Itajubá, MG, Brazil.
}

Received on 10 September, 2007

\begin{abstract}
In order to popularize the so called Schwinger's method we reconsider the Feynman propagator of two nonrelativistic systems: a charged particle in a uniform magnetic field and a charged harmonic oscillator in a uniform magnetic field. Instead of solving the Heisenberg equations for the position and the canonical momentum operators, $\mathbf{R}$ and $\mathbf{P}$, we apply this method by solving the Heisenberg equations for the gauge invariant operators $\mathbf{R}$ and $\pi=\mathbf{P}-e \mathbf{A}$, the latter being the mechanical momentum operator. In our procedure we avoid fixing the gauge from the beginning and the result thus obtained shows explicitly the gauge dependence of the Feynman propagator.
\end{abstract}

Keywords: Schwinger's method, Feynman Propagator, Magnetic Field, Harmonic Oscillator.

\section{INTRODUCTION}

In a recent paper [1], three methods were used to compute the Feynman propagators of a one-dimensional harmonic oscillator, with the purpose of allowing a student to compare the advantadges and disadvantadges of each method. The above mentioned methods were the following: the so called Schwinger's method (SM), the algebraic method and the path integral one. Though extremely powerful and elegant, Schwinger's method is by far the less popular among them. The main purpose of the present paper is to popularize Schwinger's method providing the reader with two examples slightly more difficult than the harmonic oscillator case and whose solutions may serve as a preparation for attacking relativistic problems. In some sense, this paper is complementary to reference [1].

The method we shall be concerned with was introduced by Schwinger in 1951 [2] in a paper about QED entitled "Gauge invariance and vacuum polarization". After introducing the proper time representation for computing effetive actions in QED, Schwinger was faced with a kind of non-relativistic propagator in one extra dimension. The way he solved this problem is what we mean by Schwinger's method for computing quantum propagators. For relativistic Green functions of charged particles under external electromagnetic fields, the main steps of this method are summarized in Itzykson and Zuber's textbook [3] (apart, of course, from Schwinger's work [2]). Since then, this method has been used mainly in relativistic quantum theory [4-16].

However, as mentioned before, Schwinger's method is also well suited for computing non-relativistic propagators, though it has rarely been used in this context. As far as we know, this method was used for the first time in non-relativistic quantum mechanics by Urrutia and Hernandez [17]. These authors used Schwinger's action principle to obtain the Feynman propagator for a damped harmonic oscillator with a time-dependent frequency under a time-dependent external force. Up to our knowledge, since then only a few papers have been written with this method, namely: in 1986, Urrutia and Manterola [18] used it in the problem of an anharmonic charged oscillator under a magnetic field; in the same year, Horing, Cui, and Fiorenza [19] applied Schwinger's method to obtain the Green function for crossed time-dependent electric and magnetic fields; the method was later applied in a rederivation of the Feynman propagator for a harmonic oscillator with a time-dependent frequency [20]; a connection with the midpoint-rule for path integrals involving electromagnetic interactions was discussed in [21]. Finally, pedagogical presentations of this method can be found in the recent publication [1] as well as in Schwinger's original lecture notes recently published [22], which includes a discussion of the quantum action principle and a derivation of the method to calculate propagators with some examples.

It is worth mentioning that this same method was independently developed by M. Goldberger and M. GellMann in the autumn of 1951 in connection with an unpublished paper about density matrix in statistical mechanics [23].

Our purpose in this paper is to provide the reader with two other examples of non-relativistic quantum propagators that can be computed in a straightforward way by Schwinger's method, namely: the propagator for a charged particle in a uniform magnetic field and this same problem with an additional harmonic oscillator potential. Though these problems have already been treated in the context of the quantum action principle [18], we decided to reconsider them for the following reasons: instead of solving the Heisenberg equations for the position and the canonical momentum operators, $\mathbf{R}$ and $\mathbf{P}$, as is done in [18], we apply Schwinger's method by solving the Heisenberg equations for the gauge invariant operators $\mathbf{R}$ and $\pi=\mathbf{P}-e \mathbf{A}$, the latter being the mechanical momentum operator. This is precisely the procedure followed by Schwinger in his seminal paper of gauge invariance and vacuum polarization [2]. This procedures has some nice properties. For instance, we are not obligued to choose a particular 
gauge at the beginning of calculations. As a consequence, we end up with an expression for the propagator written in an arbitrary gauge. As a bonus, the transformation law for the propagator under gauge transformations can be readly obtained.

In order to prepare the students to attack more complex problems, we solve the Heisenberg equations in matrix form, which is well suited for generalizations involving Green functions of relativistic charged particles under the influence of electromagnetic fields (constant $F_{\mu \nu}$, a plane wave field or even combinations of both). For pedagogical reasons, at the end of each calculation, we show how to extract the corresponding energy spectrum from the Feynman propagator. Although the way Schwniger's method must be applied to nonrelativistic problems has already been explained in the literature $[1,18,22]$, it is not of common knowledge so that we start this paper by summarizing its main steps. The paper is organized as follows: in the next section we review Schwinger's method, in section III we present our examples and section IV is left for the final remarks.

\section{MAIN STEPS OF SCHWINGER'S METHOD}

For simplicity, consider a one-dimensional timeindependent Hamiltonian $\mathcal{H}$ and the corresponding nonrelativistic Feynman propagator defined as

$$
K\left(x, x^{\prime} ; \tau\right)=\theta(\tau)\left\langle x\left|\exp \left[\frac{-i \mathcal{H} \tau}{\hbar}\right]\right| x^{\prime}\right\rangle,
$$

where $\theta(\tau)$ is the Heaviside step function and $|x\rangle,\left|x^{\prime}\right\rangle$ are the eingenkets of the position operator $X$ (in the Schrödinger picture) with eingenvalues $x$ and $x^{\prime}$, respectively. The extension for $3 \mathrm{D}$ systems is straightforward and will be done in the next section. For $\tau>0$ we have, from equation (1), that

$$
i \hbar \frac{\partial}{\partial \tau} K\left(x, x^{\prime} ; \tau\right)=\left\langle x\left|\mathcal{H} \exp \left[\frac{-i \mathcal{H} \tau}{\hbar}\right]\right| x^{\prime}\right\rangle .
$$

Inserting the unity $11=\exp [-(\mathrm{i} / \hbar) \mathcal{H} \tau] \exp [(\mathrm{i} / \hbar) \mathcal{H} \tau]$ in the r.h.s. of the above expression and using the well known relation between operators in the Heisenberg and Schrödinger pictures, we get the equation for the Feynman propagator in the Heisenberg picture,

$$
i \hbar \frac{\partial}{\partial \tau} K\left(x, x^{\prime} ; \tau\right)=\left\langle x, \tau|\mathcal{H}(X(0), P(0))| x^{\prime}, 0\right\rangle,
$$

where $|x, \tau\rangle$ and $\left|x^{\prime}, 0\right\rangle$ are the eingenvectors of operators $X(\tau)$ and $X(0)$, respectively, with the corresponding eingenvalues $x$ and $x^{\prime}: X(\tau)|x, \tau\rangle=x|x, \tau\rangle$ and $X(0)\left|x^{\prime}, 0\right\rangle=x^{\prime}\left|x^{\prime}, 0\right\rangle$, with $K\left(x, x^{\prime} ; \tau\right)=\left\langle x, \tau \mid x^{\prime}, 0\right\rangle$. Besides, $X(\tau)$ and $P(\tau)$ satisfy the Heisenberg equations,

$$
i \hbar \frac{d X}{d \tau}(\tau)=[X(\tau), \mathcal{H}] ; i \hbar \frac{d P}{d \tau}(\tau)=[P(\tau), \mathcal{H}]
$$

Schwinger's method consists in the following steps: (i) we solve the Heisenberg equations for $X(\tau)$ and $P(\tau)$, and write the solution for $P(0)$ only in terms of the operators $X(\tau)$ and $X(0)$;

(ii) then, we substitute the results obtained in (i) into the expression for $\mathcal{H}(X(0), P(0))$ in (3) and using the commutator $[X(0), X(\tau)]$ we rewrite each term of $\mathcal{H}$ in a time ordered form with all operators $X(\tau)$ to the left and all operators $X(0)$ to the right;

(iii) with such an ordered hamiltonian, equation (3) can be readly cast into the form

$$
i \hbar \frac{\partial}{\partial \tau} K\left(x, x^{\prime} ; \tau\right)=F\left(x, x^{\prime} ; \tau\right) K\left(x, x^{\prime} ; \tau\right),
$$

with $F\left(x, x^{\prime} ; \tau\right)$ being an ordinary function defined as

$$
F\left(x, x^{\prime} ; \tau\right)=\frac{\left\langle x, \tau\left|\mathcal{H}_{\text {ord }}(X(\tau), X(0))\right| x^{\prime}, 0\right\rangle}{\left\langle x, \tau \mid x^{\prime}, 0\right\rangle} .
$$

Integrating in $\tau$, the Feynman propagator takes the form

$$
K\left(x, x^{\prime} ; \tau\right)=C\left(x, x^{\prime}\right) \exp \left\{-\frac{i}{\hbar} \int^{\tau} F\left(x, x^{\prime} ; \tau^{\prime}\right) d \tau^{\prime}\right\},
$$

where $C\left(x, x^{\prime}\right)$ is an integration constant independent of $\tau$ and $\int^{\tau}$ means an indefinite integral;

(iv) last step is concerned with the evaluation of $C\left(x, x^{\prime}\right)$. This is done after imposing the following conditions

$$
\begin{aligned}
-i \hbar \frac{\partial}{\partial x}\left\langle x, \tau \mid x^{\prime}, 0\right\rangle & =\left\langle x, \tau|P(\tau)| x^{\prime}, 0\right\rangle, \\
i \hbar \frac{\partial}{\partial x^{\prime}}\left\langle x, \tau \mid x^{\prime}, 0\right\rangle & =\left\langle x, \tau|P(0)| x^{\prime}, 0\right\rangle,
\end{aligned}
$$

as well as the initial condition

$$
\lim _{\tau \rightarrow 0^{+}} K\left(x, x^{\prime} ; \tau\right)=\delta\left(x-x^{\prime}\right) .
$$

Imposing conditions (8) and (9) means to substitute in their left hand sides the expression for $\left\langle x, \tau \mid x^{\prime}, 0\right\rangle$ given by (7), while in their right hand sides the operators $P(\tau)$ and $P(0)$, respectively, written in terms of the operators $X(\tau)$ and $X(0)$ with the appropriate time ordering.

\section{EXAMPLES}

\section{A. Charged particle in an uniform magnetic field}

As our first example, we consider the propagator of a nonrelativistic particle with electric charge $e$ and mass $m$, submitted to a constant and uniform magnetic field B. Even though this is a genuine three-dimensional problem, the extension of the results reviewed in the last section to this case is straightforward. Since there is no electric field present, the hamiltonian can be written as

$$
\mathcal{H}=\frac{(\mathbf{P}-e \mathbf{A})^{2}}{2 m}=\frac{\pi^{2}}{2 m},
$$


where $\mathbf{P}$ is the canonical momentum operator, $\mathbf{A}$ is the vector potential and $\pi=\mathbf{P}-e \mathbf{A}$ is the gauge invariant mechanical momentum operation. We choose the axis such that the magnetic field is given by $\mathbf{B}=B \mathbf{e}_{3}$. Hence, the hamiltonian can be decomposed as

$$
\mathcal{H}=\frac{\pi_{1}^{2}+\pi_{2}^{2}}{2 m}+\frac{P_{3}^{2}}{2 m}=\mathcal{H}_{\perp}+\frac{P_{3}^{2}}{2 m},
$$

with an obvious definition for $\mathcal{H}_{\perp}$.

Since the motion along the $O \mathcal{X}_{3}$ direction is free, the threedimensional propagator $K\left(\mathbf{x}, \mathbf{x}^{\prime} ; \tau\right)$ can be written as a product of a two-dimensional propagator, $K_{\perp}\left(\mathbf{r}, \mathbf{r}^{\prime} ; \tau\right)$, related to the magnetic field and a one-dimensional free propagator, $K_{3}^{(0)}\left(x_{3}, x_{3}^{\prime} ; \tau\right)$ :

$$
K\left(\mathbf{x}, \mathbf{x}^{\prime} ; \tau\right)=K_{\perp}\left(\mathbf{r}, \mathbf{r}^{\prime} ; \tau\right) K_{3}^{(0)}\left(x_{3}, x_{3}^{\prime} ; \tau\right), \quad(\tau>0)
$$

where $\mathbf{r}=x_{1} \mathbf{e}_{1}+x_{2} \mathbf{e}_{2}$ and $K_{3}^{(0)}\left(x_{3}, x_{3}^{\prime} ; \tau\right)$ is the well known propagator of the free particle [24],

$$
K_{3}^{(0)}\left(x_{3}, x_{3}^{\prime} ; \tau\right)=\sqrt{\frac{m}{2 \pi i \hbar \tau}} \exp \left[\frac{i m}{2 \hbar} \frac{\left(x_{3}-x_{3}^{\prime}\right)^{2}}{\tau}\right] .
$$

In order to use Schwinger's method to compute the twodimensional propagator $K_{\perp}\left(\mathbf{r}, \mathbf{r}^{\prime} ; \tau\right)=\left\langle\mathbf{r}, \tau \mid \mathbf{r}^{\prime}, 0\right\rangle$, we start by writing the differential equation

$$
i \hbar \frac{\partial}{\partial \tau}\left\langle\mathbf{r}, \tau \mid \mathbf{r}^{\prime}, 0\right\rangle=\left\langle\mathbf{r}, \tau\left|\mathcal{H}_{\perp}\left(\mathbf{R}_{\perp}(0), \pi_{\perp}(0)\right)\right| \mathbf{r}^{\prime}, 0\right\rangle,
$$

where $\mathbf{R}_{\perp}(\tau)=X_{1}(\tau) \mathbf{e}_{1}+X_{2}(\tau) \mathbf{e}_{2}$ and $\pi_{\perp}(\tau)=\pi_{1}(\tau) \mathbf{e}_{\mathbf{1}}+$ $\pi_{2}(\tau) \mathbf{e}_{2}$. In (15) $|\mathbf{r}, \tau\rangle$ and $\left|\mathbf{r}^{\prime}, 0\right\rangle$ are the eigenvectors of position operators $\mathbf{R}(\tau)=X_{1}(\tau) \mathbf{e}_{1}+X_{2}(\tau) \mathbf{e}_{2}$ and $\mathbf{R}(0)=$ $X_{1}(0) \mathbf{e}_{1}+X_{2}(0) \mathbf{e}_{2}$, respectively. More especifically, operators $X_{1}(0), X_{1}(\tau), X_{2}(0)$ and $X_{2}(\tau)$ have the eigenvalues $x_{1}^{\prime}, x_{1}, x_{2}^{\prime}$ and $x_{2}$, respectively.

In order to solve the Heisenberg equations for operators $\mathbf{R}_{\perp}(\tau)$ and $\pi_{\perp}(\tau)$, we need the commutators

$$
\begin{aligned}
& {\left[X_{i}(\tau), \pi_{j}^{2}(\tau)\right]=2 i \hbar \pi_{i}(\tau),} \\
& {\left[\pi_{i}(\tau), \pi_{j}^{2}(\tau)\right]=2 i \hbar e B \varepsilon_{i j 3} \pi_{j}(\tau),}
\end{aligned}
$$

where $\varepsilon_{i j 3}$ is the usual Levi-Civita symbol. Introducing the matrix notation

$$
\mathbf{R}(\tau)=\left(\begin{array}{c}
X_{1}(\tau) \\
X_{2}(\tau)
\end{array}\right) \quad ; \Pi(\tau)=\left(\begin{array}{c}
\pi_{1}(\tau) \\
\pi_{2}(\tau)
\end{array}\right),
$$

and using the previous commutators the Heisenberg equations of motion can be cast into the form

$$
\begin{aligned}
& \frac{d \mathbf{R}(\tau)}{d \tau}=\frac{\Pi(\tau)}{m}, \\
& \frac{d \Pi(\tau)}{d \tau}=2 \omega \mathbb{C} \Pi(\tau),
\end{aligned}
$$

where $2 \omega=e B / m$ is the cyclotron frequency and we defined the anti-diagonal matrix

$$
\mathbb{C}=\left(\begin{array}{cc}
0 & 1 \\
-1 & 0
\end{array}\right)
$$

Integrating equation (19) we find

$$
\Pi(\tau)=e^{2 \omega \mathbb{C} \tau} \Pi(0) .
$$

Substituting this solution in equation (18) and integrating once more, we get

$$
\mathbf{R}(\tau)-\mathbf{R}(0)=\frac{\sin (\omega \tau)}{m \omega} e^{\omega \mathbb{C} \tau} \Pi(0),
$$

where we used the following properties of $\mathbb{C}$ matrix: $\mathbb{C}^{2}=-11 ; \mathbb{C}^{-1}=-\mathbb{C}=\mathbb{C}^{T}, e^{\alpha \mathbb{C}}=\cos (\alpha) 11+\sin (\alpha) \mathbb{C}$ with $\mathbb{C}^{T}$ being the transpose of $\mathbb{C}$. Combining equations (22) and (21) we can write $\Pi(0)$ in terms of the operators $\mathbf{R}(\tau)$ and $\mathbf{R}(0)$ as

$$
\Pi(0)=\frac{m \omega}{\sin (\omega \tau)} e^{-\omega \mathbb{C} \tau}(\mathbf{R}(\tau)-\mathbf{R}(0))
$$

In order to express $\mathcal{H}_{\perp}=\left(\pi_{1}^{2}+\pi_{2}^{2}\right) / 2 m$ in terms of $\mathbf{R}(\tau)$ and $\mathbf{R}(0)$, we use (23). In matrix notation, we have

$$
\begin{aligned}
\mathcal{H}_{\perp}=\frac{1}{2 m} \Pi^{T}(0) \Pi(0) & \\
=\frac{m \omega^{2}}{2 \sin ^{2}(\omega \tau)} & \left(\mathbf{R}^{T}(\tau) \mathbf{R}(\tau)+\mathbf{R}^{T}(0) \mathbf{R}(0)+\right. \\
& \left.-\mathbf{R}^{T}(\tau) \mathbf{R}(0)-\mathbf{R}^{T}(0) \mathbf{R}(\tau)\right) .
\end{aligned}
$$

Last term on the r.h.s. of (24) is not ordered appropriately as required in the step (ii). The correct ordering may be obtained as follows: first, we write

$$
\mathbf{R}(0)^{T} \mathbf{R}(\tau)=\mathbf{R}(\tau)^{T} \mathbf{R}(0)+\sum_{i=1}^{2}\left[X_{i}(0), X_{i}(\tau)\right] .
$$

Using equation (22), the usual commutator $\left[X_{i}(0), \pi_{j}(0)\right]=$ $i \hbar \delta_{i j} 11$ and the properties of matrix $\mathbb{C}$ it is easy to show that

$$
\sum_{i=1}^{2}\left[X_{i}(0), X_{i}(\tau)\right]=\frac{2 i \hbar \sin (\omega \tau) \cos (\omega \tau)}{m \omega},
$$

so that hamiltonian $\mathcal{H}_{\perp}$ with the appropriate time ordering takes the form

$$
\begin{aligned}
\mathcal{H}_{\perp} & =\frac{m \omega^{2}}{2 \sin ^{2}(\omega \tau)}\left\{\mathbf{R}^{2}(\tau)+\mathbf{R}^{2}(0)-2 \mathbf{R}^{T}(\tau) \mathbf{R}(0)\right\} \\
& -i \hbar \omega \cot (\omega \tau) .
\end{aligned}
$$

Substituting this hamiltonian into equation (15) and integrating in $\tau$, we obtain

$$
\left\langle\mathbf{r}, \tau \mid \mathbf{r}^{\prime}, 0\right\rangle=\frac{C\left(\mathbf{r}, \mathbf{r}^{\prime}\right)}{\sin (\omega \tau)} \exp \left\{\frac{i m \omega}{2 \hbar} \cot (\omega \tau)\left(\mathbf{r}-\mathbf{r}^{\prime}\right)^{2}\right\},
$$

where $C\left(\mathbf{r}, \mathbf{r}^{\prime}\right)$ is an integration constant to be determined by conditions (8), (9) and (10), which for the case of hand read

$$
\begin{aligned}
\left\langle\mathbf{r}, \tau\left|\pi_{j}(\tau)\right| \mathbf{r}^{\prime}, 0\right\rangle & =\left(-i \hbar \frac{\partial}{\partial x_{j}}-e A_{j}(\mathbf{r})\right)\left\langle\mathbf{r}, \tau \mid \mathbf{r}^{\prime}, 0\right\rangle \\
\left\langle\mathbf{r}, \tau\left|\pi_{j}(0)\right| \mathbf{r}^{\prime}, 0\right\rangle & =\left(i \hbar \frac{\partial}{\partial x_{j}^{\prime}}-e A_{j}\left(\mathbf{r}^{\prime}\right)\right)\left\langle\mathbf{r}, \tau \mid \mathbf{r}^{\prime}, 0\right\rangle, \\
\lim _{\tau \rightarrow 0^{+}}\left\langle\mathbf{r}, \tau \mid \mathbf{r}^{\prime}, 0\right\rangle & =\delta^{(2)}\left(\mathbf{r}-\mathbf{r}^{\prime}\right) .
\end{aligned}
$$


In order to compute the matrix element on the 1.h.s. of (29), we need to express $\Pi(\tau)$ in terms of $\mathbf{R}(\tau)$ and $\mathbf{R}(0)$. From equaitons (21) and (23), we have

$$
\Pi(\tau)=\frac{m \omega}{\sin (\omega \tau)} e^{\omega \tau \mathbb{C}}(\mathbf{R}(\tau)-\mathbf{R}(0))
$$

which leads to the matrix element

$$
\begin{aligned}
\left\langle\mathbf{r}, \tau\left|\pi_{j}(\tau)\right| \mathbf{r}^{\prime}, 0\right\rangle & =m \omega\left[\cot (\omega \tau)\left(x_{j}-x_{j}^{\prime}\right)\right. \\
& \left.+\varepsilon_{j k 3}\left(x_{k}-x_{k}^{\prime}\right)\right]\left\langle\mathbf{r}, \tau \mid \mathbf{r}^{\prime}, 0\right\rangle
\end{aligned}
$$

where we used the properties of matrix $\mathbb{C}$ and Einstein convention for repeated indices is summed. Analogously, the 1.h.s. of equation (30) can be computed from (23),

$$
\begin{aligned}
\left\langle\mathbf{r}, \tau\left|\pi_{j}(0)\right| \mathbf{r}^{\prime}, 0\right\rangle & =m \omega\left[\cot (\omega \tau)\left(x_{j}-x_{j}^{\prime}\right)\right. \\
& \left.-\varepsilon_{j k 3}\left(x_{k}-x_{k}^{\prime}\right)\right]\left\langle\mathbf{r}, \tau \mid \mathbf{r}^{\prime}, 0\right\rangle .
\end{aligned}
$$

Substituting equations (33) and (34) into (29) and (30), respectively, and using (28), we have

$$
\begin{gathered}
{\left[i \hbar \frac{\partial}{\partial x_{j}}+e A_{j}(\mathbf{r})+\frac{1}{2} e F_{j k}\left(x_{k}-x_{k}^{\prime}\right)\right] C\left(\mathbf{r}, \mathbf{r}^{\prime}\right)=0} \\
{\left[i \hbar \frac{\partial}{\partial x_{j}^{\prime}}-e A_{j}\left(\mathbf{r}^{\prime}\right)+\frac{1}{2} e F_{j k}\left(x_{k}-x_{k}^{\prime}\right)\right] C\left(\mathbf{r}, \mathbf{r}^{\prime}\right)=0}
\end{gathered}
$$

where we defined $F_{j k}=\varepsilon_{j k 3} B$.

Our strategy to solve the above system of differential equations is the following: we first equation (35) assuming in this equation variables $\mathbf{r}^{\prime}$ as constants. Then, we impose that the result thus obtained is a solution of equation (36). With this goal, we multiply both sides of (35) by $d x_{j}$ and sum over $j$, to obtain

$$
\frac{1}{C}\left(\frac{\partial C}{\partial x_{j}} d x_{j}\right)=\frac{i e}{\hbar}\left[A_{j}(\mathbf{r})+\frac{1}{2} F_{j k}\left(x_{k}-x_{k}^{\prime}\right)\right] d x_{j} .
$$

Integration of the previous equation leads to

$$
C\left(\mathbf{r}, \mathbf{r}^{\prime}\right)=C\left(\mathbf{r}^{\prime}, \mathbf{r}^{\prime}\right) e^{\left\{\frac{i e}{\hbar} \int_{\Gamma}^{\mathbf{r}^{\prime}}\left[A_{j}\left(\xi_{j}\right)+\frac{1}{2} F_{j k}\left(\xi_{k}-x_{k}^{\prime}\right)\right] d \xi_{j}\right\}}
$$

where the line integral is assumed to be along curve $\Gamma$, to be specified in a moment. As we shall see, this line integral does not depend on the curve $\Gamma$ joining $\mathbf{r}^{\prime}$ and $\mathbf{r}$, as expected, since the 1.h.s. of (37) is an exact differencial.

In order to determine the differential equation for $C\left(\mathbf{r}^{\prime}, \mathbf{r}^{\prime}\right)$ we must substitue expression (38) into equation (36). Doing that and using carefully the fundamental theorem of differential calculus, it is straightforward to show that

$$
\frac{\partial C}{\partial x_{j}^{\prime}}\left(\mathbf{r}^{\prime}, \mathbf{r}^{\prime}\right)=0
$$

which means that $C\left(\mathbf{r}^{\prime}, \mathbf{r}^{\prime}\right)$ is a constant, $C_{0}$, independent of $\mathbf{r}^{\prime}$. Noting that

$$
\left[\mathbf{B} \times\left(\xi-\mathbf{r}^{\prime}\right)\right]_{j}=-F_{j k}\left(\xi_{k}-x_{k}^{\prime}\right),
$$

equation (38) can be written as

$$
C\left(\mathbf{r}, \mathbf{r}^{\prime}\right)=C_{0} \exp \left\{\frac{i e}{\hbar} \int_{\Gamma} \int_{\mathbf{r}^{\prime}}^{\mathbf{r}}\left[\mathbf{A}(\xi)-\frac{1}{2} \mathbf{B} \times\left(\xi-\mathbf{r}^{\prime}\right)\right] \cdot d \xi\right\} .
$$

Observe, now, that the integrand in the previous equation has a vanishing curl,

$$
\nabla_{\xi} \times\left[\mathbf{A}(\xi)-\frac{1}{2} \mathbf{B} \times\left(\xi-\mathbf{r}^{\prime}\right)\right]=\mathbf{B}-\mathbf{B}=\mathbf{0},
$$

which means that the line integral in (41) is path independent. Choosing, for convenience, the straightline from $\mathbf{r}^{\prime}$ to $\mathbf{r}$, it can be readly shown that

$$
\Gamma_{s l} \int_{\mathbf{r}^{\prime}}^{\mathbf{r}}\left[\mathbf{B} \times\left(\xi-\mathbf{r}^{\prime}\right)\right] \cdot d \xi=0,
$$

where $\Gamma_{s l}$ means a straightline from $\mathbf{r}^{\prime}$ to $\mathbf{r}$. With this simplification, the $C\left(\mathbf{r}^{\prime}, \mathbf{r}\right)$ takes the form

$$
C\left(\mathbf{r}, \mathbf{r}^{\prime}\right)=C_{0} \exp \left\{\frac{i e}{\hbar} \int_{\Gamma_{s l}} \int_{\mathbf{r}^{\prime}}^{\mathbf{r}} \mathbf{A}(\xi) \cdot d \xi\right\} .
$$

Substituting last equation into (28) and using the initial condition (10), we readly obtain $C_{0}=\frac{m \omega}{2 \pi i \hbar}$. Therefore the complete Feynman propagator for a charged particle under the influence of a constant and uniform magnetic field takes the form

$$
\begin{array}{r}
K\left(\mathbf{x}, \mathbf{x}^{\prime} ; \tau\right) \\
=\frac{m \omega}{2 \pi i \hbar \sin (\omega \tau)} \sqrt{\frac{m}{2 \pi i \hbar \tau}} \exp \left\{\frac{i e}{\hbar} \int_{\mathbf{r}^{\prime}}^{\mathbf{r}} \mathbf{A}(\xi) \cdot d \xi\right\} \\
\exp \left\{\frac{i m}{2 \hbar} \cot (\omega \tau)\left(\mathbf{r}-\mathbf{r}^{\prime}\right)^{2}\right\} \exp \left\{\frac{i m}{2 \hbar} \frac{\left(x_{3}-x_{3}^{\prime}\right)^{2}}{\tau}\right\},
\end{array}
$$

where in the above equation we omitted the symbol $\Gamma_{s l}$ but, of course, it is implicit that the line integral must be done along a straightline, and we brought back the free propagation along the $O X_{3}$ direction. A few comments about the above result are in order.

1. Firstly, we should emphasize that the line integral which appears in the first exponencial on the r.h.s. of (43) must be evaluated along a straight line between $\mathbf{r}^{\prime}$ and r. If for some reason we want to choose another path, instead of integral $\int_{\mathbf{r}^{\prime}}^{\mathbf{r}} \mathbf{A}(\xi) \cdot d \xi$, we must evaluate $\int_{\mathbf{r}^{\prime}}^{\mathbf{r}}\left[\mathbf{A}(\xi)-(1 / 2) \mathbf{B} \times\left(\xi-\mathbf{r}^{\prime}\right)\right] \cdot d \xi$.

2. Since we solved the Heisenberg equations for the gauge invariant operators $\mathbf{R}_{\perp}$ and $\pi_{\perp}$, our final result is written for a generic gauge. Note that the gauge-independent and gauge-dependent parts of the propagator are clearly separated. The gauge fixing corresponds to choose a particular expression for $\mathbf{A}(\xi)$. Besides, from (43) we imediately obtain the transformation law for the propagator under a gauge transformation $\mathbf{A} \rightarrow \mathbf{A}+\nabla \Lambda$, namely,

$$
K\left(\mathbf{r}, \mathbf{r}^{\prime} ; \tau\right) \longmapsto e^{\frac{i e}{\hbar} \Lambda(\mathbf{r})} K\left(\mathbf{r}, \mathbf{r}^{\prime} ; \tau\right) e^{-\frac{i e}{\hbar} \Lambda\left(\mathbf{r}^{\prime}\right)}
$$

Although this transformation law was obtained in a particular case, it can be shown that it is quite general. 
3. It is interesting to show how the energy spectrum (Landau levels), with the corresponding degeneracy per unit area, can be extracted from propagator (43). With this purpose, we recall that the partition function can be obtained from the Feynman propagator by taking $\tau=-i \hbar \beta$, with $\beta=1 /\left(K_{B} T\right)$, and taking the spatial trace,

$$
Z(\beta)=\int_{-\infty}^{\infty} d x_{1} \int_{-\infty}^{\infty} d x_{2} K(\mathbf{r}, \mathbf{r} ;-i \hbar \beta)
$$

Substituting (43) into last expression, we get

$$
Z(\beta)=\int_{-\infty}^{\infty} d x_{1} \int_{-\infty}^{\infty} d x_{2} \frac{m \omega}{2 \pi \hbar \operatorname{senh}(\hbar \beta \omega)},
$$

where we used the fact that $\sin (-i \theta)=-i \sinh \theta$. Observe that the above result is divergent, since the area of the $O X_{1} X_{2}$ plane is infinite. This is a consequence of the fact that each Landau level is infinitely degenerated, though the degeneracy per unit area is finite. In order to proceed, let us assume an area as big as we want, but finite. Adopting this kind or regularization, we write

$$
\begin{array}{rl}
\int_{-L / 2}^{L / 2} d x_{1} \int_{-L / 2}^{L / 2} d x_{2} & K(\mathbf{r}, \mathbf{r} ;-i \hbar \beta) \approx \frac{L^{2} m \omega}{2 \pi \hbar \operatorname{senh}(\hbar \beta \omega)} \\
& =\frac{L^{2} e B}{2 \pi \hbar\left(e^{\hbar \beta \omega}-e^{-\hbar \beta \omega}\right)} \\
& =\frac{L^{2} e B}{2 \pi \hbar} \frac{e^{-\frac{1}{2} \hbar \beta \omega_{c}}}{\left(1-e^{-\hbar \beta \omega_{c}}\right)} \\
& =\sum_{n=0}^{\infty} \frac{L^{2} e B}{2 \pi \hbar} e^{-\beta\left(n+\frac{1}{2}\right) \hbar \omega_{c}}
\end{array}
$$

where we denoted by $\omega_{c}=e B / 2 m$ the ciclotron frequency. Comparing this result with that of a partition function whose energy level $E_{n}$ has degeneracy $g_{n}$, given by

$$
Z(\beta)=\sum_{n} g_{n} e^{-\beta E_{n}}
$$

we imediately identify the so called Landau leves and the corresponding degeneracy per unit area,

$$
E_{n}=\left(n+\frac{1}{2}\right) \hbar \omega_{c} ; \frac{g_{n}}{A}=\frac{e B}{2 \pi \hbar} \quad(n=0,1, \ldots)
$$

\section{B. Charged harmonic oscillator in a uniform magnetic field}

In this section we consider a particle with mass $m$ and charge $e$ in the presence of a constant and uniform magnetic field $\mathbf{B}=B \mathbf{e}_{3}$ and submitted to a 2-dimensional isotropic harmonic oscillator potential in the $O X_{1} X_{2}$ plane, with natural frequency $\omega_{0}$. Using the same notation as before, we can write the hamiltonian of the system in the form

$$
\mathcal{H}=\mathcal{H}_{\perp}+\frac{P_{3}^{2}}{2 m},
$$

where

$$
\mathcal{H}_{\perp}=\frac{\pi_{1}^{2}+\pi_{2}^{2}}{2 m}+\frac{1}{2} m \omega_{0}^{2}\left(X_{1}^{2}+X_{2}^{2}\right) .
$$

As before, the Feynman propagator for this problem takes the form $K\left(\mathbf{x}, \mathbf{x}^{\prime} ; \tau\right)=K_{\perp}\left(\mathbf{r}, \mathbf{r}^{\prime} ; \tau\right) K_{3}^{(0)}\left(x_{3}, x_{3}^{\prime} ; \tau\right)$, with $K_{3}^{(0)}\left(x_{3}, x_{3}^{\prime} ; \tau\right)$ given by equation (14). The propagator in the $O X_{1} X_{2}$-plane satisfies the differential equation (15) and will be determined by the same used in the previous example.

Using hamiltonian (47) and the usual commutation relations the Heisenberg equations are given by

$$
\begin{aligned}
& \frac{d \mathbf{R}(\tau)}{d \tau}=\frac{\Pi(\tau)}{m}, \\
& \frac{d \Pi(\tau)}{d \tau}=2 \omega \mathbb{C} \Pi(\tau)-m \omega_{0}^{2} \mathbf{R}(\tau),
\end{aligned}
$$

where we have used the matrix notation introduced in (17) and (20). Equation (48) is the same as (18), but equation (49) contains an extra term when compared to (19). In order to decouple equations (48) and (49), we differentiate (48) with respect to $\tau$ and then use (49). This procedure leads to the following uncoupled equation

$$
\frac{d^{2} \mathbf{R}(\tau)}{d \tau^{2}}-2 \omega \mathbb{C} \frac{d \mathbf{R}(\tau)}{d \tau}+\omega_{0}^{2} \mathbf{R}(\tau)=0
$$

After solving this equation, $\mathbf{R}(\tau)$ and $\Pi(\tau)$ are constrained to satisfy equations (48) and (49), respectively. A straightforward algebra yields the solution

$$
\begin{aligned}
& \mathbf{R}(\tau)=\mathbb{M}^{-} \mathbf{R}(0)+\mathbb{N} \Pi(0) \\
& \Pi(\tau)=\mathbb{M}^{+} \Pi(0)-m^{2} \omega_{0}^{2} \mathbb{N R}(0),
\end{aligned}
$$

where we defined the matrices

$$
\begin{aligned}
\mathbb{N} & =\frac{\sin (\Omega \tau)}{m \Omega} e^{\omega \tau \mathbb{C}} \\
\mathbb{M}^{ \pm} & =e^{\omega \tau \mathbb{C}}\left[\cos (\Omega \tau) 11 \pm \frac{\omega}{\Omega} \sin (\Omega \tau) \mathbb{C}\right]
\end{aligned}
$$

and frequency $\Omega=\sqrt{\omega^{2}+\omega_{0}^{2}}$. Using (51) and (52), we write $\Pi(0)$ and $\Pi(\tau)$ in terms of $\mathbf{R}(\tau)$ and $\mathbf{R}(0)$,

$$
\begin{aligned}
& \Pi(0)=\mathbb{N}^{-1} \mathbf{R}(\tau)-\mathbb{N}^{-1} \mathbb{M}^{-} \mathbf{R}(0) \\
& \Pi(\tau)=\mathbb{M}^{+} \mathbb{N}^{-1} \mathbf{R}(\tau)-\left[\mathbb{M}^{+} \mathbb{N}^{-1} \mathbb{M}^{-}+m^{2} \omega_{0}^{2} \mathbb{N}\right] \mathbf{R}(0) .
\end{aligned}
$$

Now, we must order appropriately the hamiltonian operator $\mathcal{H}_{\perp}=\Pi^{T}(0) \Pi(0) /(2 m)+m \omega_{0}^{2} \mathbf{R}^{T}(0) \mathbf{R}(0) / 2$, which, with the aid of equation (55), can be written as

$$
\mathcal{H}_{\perp}=\left[\mathbf{R}^{T}(\tau)\left(\mathbb{N}^{-1}\right)^{T}-\mathbf{R}^{T}(0)\left(\mathbb{M}^{-}\right)^{T}\left(\mathbb{N}^{-1}\right)^{T}\right]
$$




$$
\begin{aligned}
& \times\left[\mathbb{N}^{-1} \mathbf{R}(\tau)-\mathbb{N}^{-1} \mathbb{M}^{-} \mathbf{R}(0)\right]+m \omega_{0}^{2} \mathbf{R}^{T}(0) \mathbf{R}(0) \\
&= \frac{m \Omega^{2}}{2 \sin ^{2}(\Omega \tau)}\left[\mathbf{R}^{T}(\tau)-\mathbf{R}^{T}(0)\left(\mathbb{M}^{-}\right)^{T}\right] \\
& \times\left[\mathbf{R}(\tau)-\mathbb{M}^{-} \mathbf{R}(0)\right]+m \omega_{0}^{2} \mathbf{R}^{T}(0) \mathbf{R}(0) \\
&= \frac{m \Omega^{2}}{2 \sin ^{2}(\Omega \tau)}\left[\mathbf{R}^{T}(\tau) \mathbf{R}(\tau)-\mathbf{R}^{T}(\tau) \mathbb{M}^{-} \mathbf{R}(0)\right. \\
&\left.-\mathbf{R}^{T}(0)\left(\mathbb{M}^{-}\right)^{T} \mathbf{R}^{T}(\tau)+\mathbf{R}^{T}(0)\left(\mathbb{M}^{-}\right)^{T} \mathbb{M}^{-} \mathbf{R}(0)\right] \\
&+m \omega_{0}^{2} \mathbf{R}^{T}(0) \mathbf{R}(0) \\
&= \frac{m \Omega^{2}}{2 \sin ^{2}(\Omega \tau)}\left[\mathbf{R}^{2}(\tau)-\mathbf{R}^{T}(\tau) \mathbb{M}^{-} \mathbf{R}(0)\right. \\
&\left.\quad-\mathbf{R}^{T}(0)\left(\mathbb{M}^{-}\right)^{T} \mathbf{R}(\tau)+\mathbf{R}^{2}(0)\right]
\end{aligned}
$$

where superscript $T$ means transpose and we have used the properties of the matrices $\mathbb{N}$ and $\mathbb{M}^{-}$given by (53) and (54). In order to get the right time ordering, observe first that

$\mathbf{R}^{T}(0)\left(\mathbb{M}^{-}\right)^{T} \mathbf{R}(\tau)=\mathbf{R}^{T}(\tau) \mathbb{M}^{-} \mathbf{R}(0)+\left[\left(\mathbb{M}^{-} \mathbf{R}(0)\right)_{i}, \mathbf{X}_{i}(\tau)\right]$, where

$$
\left[\left(\mathbb{M}^{-} \mathbf{R}(0)\right)_{i}, \mathbf{X}_{i}(\tau)\right]=i \hbar \operatorname{Tr}\left[\mathbb{N}\left(\mathbb{M}^{-}\right)^{T}\right]=\frac{i \hbar}{m \Omega} \sin (2 \Omega \tau) .
$$

Using the last two equations into (57) we rewrite the hamiltonian in the desired ordered form, namely,

$$
\begin{gathered}
\mathcal{H}_{\perp}=\frac{m \Omega^{2}}{2 \sin ^{2}(\Omega \tau)}\left[\mathbf{R}^{2}(\tau)+\mathbf{R}^{2}(0)-2 \mathbf{R}^{T}(\tau) \mathbb{M}^{-} \mathbf{R}(0)\right. \\
\left.-\frac{i \hbar}{m \Omega} \sin (2 \Omega \tau)\right] .
\end{gathered}
$$

For future convenience, let us define

$$
\begin{aligned}
& U(\tau)=\cos (\omega \tau) \cos (\Omega \tau)+\frac{\omega}{\Omega} \sin (\omega \tau) \sin (\Omega \tau), \\
& V(\tau)=\sin (\omega \tau) \cos (\Omega \tau)-\frac{\omega}{\Omega} \cos (\omega \tau) \sin (\Omega \tau)
\end{aligned}
$$

and write matrix $\mathbb{M}^{-}$, defined in (54), in the form

$$
\mathbb{M}^{-}=U(\tau) 11+\mathrm{V}(\tau) \mathbb{C}
$$

Substituting (61) in (58) we have

$$
\begin{aligned}
\mathcal{H}_{\perp} & =\frac{m \Omega^{2}}{2 \sin ^{2}(\Omega \tau)}\left[\mathbf{R}^{2}(\tau)+\mathbf{R}^{2}(0)-2 U(\tau) \mathbf{R}^{T}(\tau) \mathbf{R}(0)\right. \\
& \left.-2 V(\tau) \mathbf{R}^{T}(\tau) \mathbb{C} \mathbf{R}(0)-\frac{i \hbar}{m \Omega} \sin (2 \Omega \tau)\right] .
\end{aligned}
$$

The next step is to compute the classical function $F\left(\mathbf{r}, \mathbf{r}^{\prime} ; \tau\right)$. Using the following identities

$$
\begin{aligned}
& \frac{\Omega U(\tau)}{\sin ^{2}(\Omega \tau)}=-\frac{d}{d \tau}\left[\frac{\cos (\omega \tau)}{\sin (\Omega \tau)}\right], \\
& \frac{\Omega V(\tau)}{\sin ^{2}(\Omega \tau)}=-\frac{d}{d \tau}\left[\frac{\sin (\omega \tau)}{\sin (\Omega \tau)}\right],
\end{aligned}
$$

into (62), we write $F\left(\mathbf{r}, \mathbf{r}^{\prime} ; \tau\right)$ in the convenient form

$$
\begin{aligned}
F\left(\mathbf{r}, \mathbf{r}^{\prime} ; \tau\right) & =\frac{m \Omega^{2}}{2}\left(\mathbf{r}^{2}+\mathbf{r}^{\prime^{2}}\right) \csc (\Omega \tau)^{2}+m \Omega \mathbf{r} \cdot \mathbf{r}^{\prime} \frac{d}{d \tau}\left[\frac{\cos (\omega \tau)}{\sin (\Omega \tau)}\right] \\
& +m \Omega \mathbf{r} \cdot \mathbb{C} \mathbf{r}^{\prime} \frac{d}{d \tau}\left[\frac{\sin (\omega \tau)}{\sin (\Omega \tau)}\right]-i \hbar \Omega \frac{\cos (\Omega \tau)}{\sin (\Omega \tau)} .
\end{aligned}
$$

Inserting this result into the differential equation

$$
i \hbar \frac{\partial}{\partial \tau}\left\langle\mathbf{r}, \tau \mid \mathbf{r}^{\prime}, 0\right\rangle=F\left(\mathbf{r}, \mathbf{r}^{\prime} ; \tau\right)\left\langle\mathbf{r}, \tau \mid \mathbf{r}^{\prime}, 0\right\rangle,
$$

and integrating in $\tau$, we obtain

$$
\begin{gathered}
\left\langle\mathbf{r}, \tau \mid \mathbf{r}^{\prime}, 0\right\rangle=\frac{C\left(\mathbf{r}, \mathbf{r}^{\prime}\right)}{\sin (\Omega \tau)} \exp \left\{\frac { i m \Omega } { 2 \hbar } \left[\left(\mathbf{r}^{2}+\mathbf{r}^{\prime^{2}}\right) \cot (\Omega \tau)\right.\right. \\
\left.\left.-2\left(\mathbf{r} \cdot \mathbf{r}^{\prime} \frac{\cos (\omega \tau)}{\sin (\Omega \tau)}+\mathbf{r} \cdot \mathbb{C} \mathbf{r}^{\prime} \frac{\sin (\omega \tau)}{\sin (\Omega \tau)}\right)\right]\right\} .
\end{gathered}
$$

where $C\left(\mathbf{r}, \mathbf{r}^{\prime}\right)$ is an arbitrary integration constantto be determined by conditions (29), (30) and (31). Using (56) we can calculate the 1.h.s. of condition (29),

$$
\begin{aligned}
& \left\langle\mathbf{r}, \tau\left|\pi_{j}(\tau)\right| \mathbf{r}^{\prime}, 0\right\rangle=\frac{m \Omega}{\sin (\Omega \tau)}\left\{\cos (\Omega \tau) x_{j}-\cos (\omega \tau) x_{j}^{\prime}\right. \\
& \left.+\left[\frac{\omega}{\Omega} \sin (\Omega \tau) x_{k}-\sin (\omega \tau) x_{k}^{\prime}\right] \varepsilon_{j k 3}\right\}\left\langle\mathbf{r}, \tau \mid \mathbf{r}^{\prime}, 0\right\rangle,
\end{aligned}
$$

and using (55) we get the 1.h.s. of condition (30),

$$
\begin{aligned}
& \left\langle\mathbf{r}, \tau\left|\pi_{j}(0)\right| \mathbf{r}^{\prime}, 0\right\rangle=\frac{m \Omega}{\sin (\Omega \tau)}\left\{\cos (\omega \tau) x_{j}-\cos (\Omega \tau) x_{j}^{\prime}\right. \\
& \left.+\left[\frac{\omega}{\Omega} \sin (\Omega \tau) x_{k}^{\prime}-\sin (\omega \tau) x_{k}\right] \varepsilon_{j k 3}\right\}\left\langle\mathbf{r}, \tau \mid \mathbf{r}^{\prime}, 0\right\rangle .
\end{aligned}
$$

With the help of the simple identities

$$
\begin{array}{cc}
\frac{\partial}{\partial x_{j}}\left(\mathbf{r}^{2}+\mathbf{r}^{\prime^{2}}\right)=2 x_{j} & ; \quad \frac{\partial}{\partial x_{j}^{\prime}}\left(\mathbf{r}^{2}+\mathbf{r}^{\prime 2}\right)=2 x_{j}^{\prime} \\
\frac{\partial}{\partial x_{j}} \mathbf{r} \cdot \mathbf{r}^{\prime}=x_{j}^{\prime} \quad ; \quad \frac{\partial}{\partial x_{j}^{\prime}} \mathbf{r} \cdot \mathbf{r}^{\prime}=x_{j} \\
\frac{\partial}{\partial x_{j}} \mathbf{r} \cdot \mathbb{C} \mathbf{r}^{\prime}=\varepsilon_{j k 3} x_{k}^{\prime} \quad ; \quad \frac{\partial}{\partial x_{j}^{\prime}} \mathbf{r} \cdot \mathbb{C} \mathbf{r}^{\prime}=-\varepsilon_{j k 3} x_{k} .
\end{array}
$$

and also using equation (66), we are able to compute the right hand sides of conditions (29) and (30), which are given, respectively, by

$$
\begin{aligned}
& \left\{-\frac{i \hbar}{C\left(\mathbf{r}, \mathbf{r}^{\prime}\right)} \frac{\partial C\left(\mathbf{r}, \mathbf{r}^{\prime}\right)}{\partial x_{j}}+m \Omega \frac{\cos (\Omega \tau)}{\sin (\Omega \tau)} x_{j}-m \Omega \frac{\cos (\omega \tau)}{\sin (\Omega \tau)} x_{j}^{\prime}\right. \\
& \left.-m \Omega \frac{\sin (\omega \tau)}{\sin (\Omega \tau)} \varepsilon_{j k 3} x_{k}^{\prime}-e A_{j}(\mathbf{r})\right\}\left\langle\mathbf{r}, \tau \mid \mathbf{r}^{\prime}, 0\right\rangle
\end{aligned}
$$

and

$$
\begin{aligned}
& \left\{\quad \frac{i \hbar}{C\left(\mathbf{r}, \mathbf{r}^{\prime}\right)} \frac{\partial C\left(\mathbf{r}, \mathbf{r}^{\prime}\right)}{\partial x_{j}^{\prime}} m \Omega \frac{\cos (\Omega \tau)}{\sin (\Omega \tau)} x_{j}^{\prime}+m \Omega \frac{\cos (\omega \tau)}{\sin (\Omega \tau)} x_{j}\right. \\
& \left.-m \Omega \frac{\sin (\omega \tau)}{\sin (\Omega \tau)} \varepsilon_{j k 3} x_{k}-e A_{j}\left(\mathbf{r}^{\prime}\right)\right\}\left\langle\mathbf{r}, \tau \mid \mathbf{r}^{\prime}, 0\right\rangle .
\end{aligned}
$$


Equating (67) and (69), and also (68) and (70)), we get the system of differential equations for $C\left(\mathbf{r}, \mathbf{r}^{\prime}\right)$

$$
\begin{aligned}
& i \hbar \frac{\partial C\left(\mathbf{r}, \mathbf{r}^{\prime}\right)}{\partial x_{j}}+e\left[A_{j}(\mathbf{r})+\frac{F_{j k}}{2} x_{k}\right] C\left(\mathbf{r}, \mathbf{r}^{\prime}\right)=0 \\
& i \hbar \frac{\partial C\left(\mathbf{r}, \mathbf{r}^{\prime}\right)}{\partial x_{j}^{\prime}}-e\left[A_{j}\left(\mathbf{r}^{\prime}\right)+\frac{F_{j k}}{2} x_{k}^{\prime}\right] C\left(\mathbf{r}, \mathbf{r}^{\prime}\right)=0 .
\end{aligned}
$$

Proceeding as in the previous example, we first integrate (71). With this goal, we multiply it by $d x_{j}$, sum in $j$ and integrate it to obtain

$$
C\left(\mathbf{r}, \mathbf{r}^{\prime}\right)=C\left(\mathbf{r}^{\prime}, \mathbf{r}^{\prime}\right) \exp \left\{\frac{i e}{\hbar} \int_{\Gamma}^{\mathbf{r}^{\prime}}{ }^{\mathbf{r}}\left[A_{j}(\xi)+\frac{F_{j k}}{2} \xi_{k}\right] d \xi_{j}\right\},
$$

where the path of integration $\Gamma$ will be specified in a moment. Inserting expression (73) into the second differential equation (72), we get

$$
\frac{\partial}{\partial x_{j}^{\prime}} C\left(\mathbf{r}^{\prime}, \mathbf{r}^{\prime}\right)=0 \Longrightarrow C\left(\mathbf{r}^{\prime}, \mathbf{r}^{\prime}\right)=C_{0}
$$

where $C_{0}$ is a constant independent of $\mathbf{r}^{\prime}$, so that equation (73) can be cast, after some convenient rearrangements, into the form

$$
C\left(\mathbf{r}, \mathbf{r}^{\prime}\right)=C_{0} \exp \left\{\frac{i e}{\hbar} \int_{\mathbf{r}^{\prime}}^{\mathbf{r}}\left[\mathbf{A}(\xi)-\frac{1}{2} \mathbf{B} \times \xi\right] \cdot d \xi\right\} .
$$

Note that the integrand has a vanishing curl so that we can choose the path of integration $\Gamma$ at our will. Choosing, as before, the straight line between $\mathbf{r}^{\prime}$ and $\mathbf{r}$, it can be shown that

$$
\int_{\mathbf{r}^{\prime}}^{\mathbf{r}}\left[\mathbf{A}(\xi)-\frac{\mathbf{B}}{2} \times \xi\right] \cdot d \xi=\int_{\mathbf{r}^{\prime}}^{\mathbf{r}} \mathbf{A}(\xi) \cdot d \xi+\frac{1}{2} B \mathbf{r} \cdot \mathbb{C} \mathbf{r}^{\prime}
$$

where, for simplicity of notation, we omitted the symbol $\Gamma_{s l}$ indicating that the line integral must be done along a straight line. From equations (73), (74) e (75), we get

$$
C\left(\mathbf{r}, \mathbf{r}^{\prime}\right)=C_{0} \exp \left\{\frac{i e}{\hbar} \int_{\mathbf{r}^{\prime}}^{\mathbf{r}} \mathbf{A}(\xi) \cdot d \xi\right\} \exp \left\{\frac{i m}{\hbar} \mathbf{r} \cdot \mathbb{C} \mathbf{r}^{\prime}\right\}
$$

which substituted back into equation (66) yields

$$
\begin{array}{r}
\left\langle\mathbf{r}, \tau \mid \mathbf{r}^{\prime}, 0\right\rangle=\frac{C_{0}}{\sin (\Omega \tau)} \exp \left\{\frac{i e}{\hbar} \int_{\mathbf{r}^{\prime}}^{\mathbf{r}} \mathbf{A}(\xi) \cdot d \xi\right\} \\
\exp \left\{\frac { i m \Omega } { 2 \hbar \operatorname { s i n } ( \Omega \tau ) } \left\{\left(\mathbf{r}^{2}+\mathbf{r}^{\prime^{2}}\right) \cos (\Omega \tau)\right.\right. \\
\left.\left.-2 \mathbf{r} \cdot \mathbf{r}^{\prime} \cos (\omega \tau)-2\left[\sin (\omega \tau)-\frac{\omega}{\Omega} \sin \Omega \tau\right] \mathbf{r} \mathbb{C} \mathbf{r}^{\prime}\right\}\right\}
\end{array}
$$

The initial condition implies $C_{0}=m \Omega /(2 \pi i \hbar)$. Hence, the desired Feynman propagator is finally given by

$$
\begin{aligned}
K\left(\mathbf{x}, \mathbf{x}^{\prime} ; \tau\right)= & K_{\perp}\left(\mathbf{r}, \mathbf{r}^{\prime} ; \tau\right) K_{3}^{(0)}\left(x_{3}, x_{3}^{\prime} ; \tau\right) \\
= & \frac{m \Omega}{2 \pi i \hbar \sin (\Omega \tau)} \sqrt{\frac{m}{2 \pi i \hbar \tau}} \exp \left\{\frac{i e}{\hbar} \int_{\mathbf{r}^{\prime}}^{\mathbf{r}} \mathbf{A}(\xi) \cdot d \xi\right\} \exp \left\{\frac { i m \Omega } { 2 \hbar \operatorname { s i n } ( \Omega \tau ) } \left\{\cos (\Omega \tau)\left(\mathbf{r}^{2}+\mathbf{r}^{\prime^{2}}\right)\right.\right. \\
& \left.\left.-2 \cos (\omega \tau) \mathbf{r} \cdot \mathbf{r}^{\prime}-2\left[\sin (\omega \tau)-\frac{\omega}{\Omega} \sin (\Omega \tau)\right] \mathbf{r} \cdot \mathbb{C} \mathbf{r}^{\prime}\right\}\right\} \exp \left\{\frac{i m}{2 \hbar} \frac{\left(x_{3}-x_{3}^{\prime}\right)^{2}}{\tau}\right\},
\end{aligned}
$$

where we brought back the free part of the propagator corresponding to the movement along the $O X_{3}$ direction. Of course, for $\omega_{0}=0$ we reobtain the propagator found in our first example and for $\mathbf{B}=\mathbf{0}$ we reobtain the propagator for a bidimensional oscillator in the $O X_{1} X_{2}$ plane multiplied by a free propagator in the $O x_{3}$ direction, as can be easily checked.

Regarding the gauge dependence of the propagator, the same comments done before are still valid here, namely, the above expression is written for a generic gauge, the transformation law for the propagator under a gauge transformation is the same as before, etc. We finish this section, extracting from the previous propagator, the corresponding energy spectrum. With this purpose, we first compute the trace of the propagator,

$$
\begin{aligned}
\int_{-\infty}^{\infty} d x_{1} \int_{-\infty}^{\infty} d x_{2} K_{\perp}^{\prime}\left(x_{1}, x_{1}, x_{2}, x_{2} ; \tau\right) & =\frac{m \Omega}{2 \pi i \hbar \sin (\Omega \tau)} \int_{-\infty}^{\infty} d x_{1} \int_{-\infty}^{\infty} d x_{2} \exp \left\{\frac{i m \Omega}{2 \hbar \sin (\Omega \tau)}\left[2(\cos (\Omega \tau)-\cos (\omega \tau))\left(x_{1}^{2}+x_{2}^{2}\right)\right]\right\} \\
& =\frac{1}{2[\cos (\Omega \tau)-\cos (\omega \tau)]}
\end{aligned}
$$

where we used the well known result for the Fresnel integral.

Using now the identity

$$
\cos (\Omega \tau)-\cos (\omega \tau)=-2 \sin [(\Omega+\omega) \tau / 2] \sin [(\Omega-\omega) \tau / 2)],
$$


we get for the corresponding energy Green function

$$
\begin{aligned}
& \mathcal{G}(E)=-i \int_{0}^{\infty} d \tau e^{\frac{i}{\hbar} E \tau} \int_{-\infty}^{\infty} d x_{1} \int_{-\infty}^{\infty} d x_{2} K_{\perp}^{\prime}\left(x_{1}, x_{1}, x_{2}, x_{2} ; \tau\right) \\
& =\frac{i}{4} \int_{0}^{\infty} d \tau \frac{e^{\frac{i}{\hbar} E \tau}}{\operatorname{sen}\left(\frac{\Omega+\omega}{2} \tau\right) \operatorname{sen}\left(\frac{\Omega-\omega}{2} \tau\right)} \\
& =-i \int_{0}^{\infty} d \tau e^{\frac{i}{\hbar} E \tau}\left(\sum_{l=0}^{\infty} e^{-\left(l+\frac{1}{2}\right)(\Omega+\omega) \tau}\right)\left(\sum_{n=0}^{\infty} e^{-i\left(n+\frac{1}{2}\right)(\Omega-\omega) \tau}\right) \text {, }
\end{aligned}
$$

where is tacitly assumed that $E \rightarrow E-i \varepsilon$ and we also used that (with the assumption $v \rightarrow v-i \varepsilon$ )

$$
\frac{1}{\operatorname{sen}\left(\frac{v}{2} \tau\right)}=2 i \sum_{n-0}^{\infty} e^{-i\left(n+\frac{1}{2}\right) v \tau} \text {. }
$$

Changing the order of integration and summations, and integrating in $\tau$, we finally obtain

$$
\mathcal{G}(E)=\sum_{l, n=0}^{\infty} \frac{1}{E-E_{n l}},
$$

where the poles of $\mathcal{G}(E)$, which give the desired energy levels, are identified as

$$
E_{n l}=(l+n+1) \hbar \Omega+(l-n) \hbar \omega, \quad(l, n=0,1, \ldots) .
$$

The Landau levels can be reobtained from the previous result by simply taking the limit $\omega_{0} \rightarrow 0$ :

$$
E_{n l} \longrightarrow(2 l+1) \hbar \omega=\left(l+\frac{1}{2}\right) \hbar \omega_{c},
$$

with $l=0,1, \ldots$ and $\omega_{c}=e B / m$, in agreement to the result we had already obtained before.

\section{FINAL REMARKS}

In this paper we reconsidered, in the context of Schwinger's method, the Feynman propagators of two well known problems, namely, a charged particle under the influence of a constant and uniform magnetic field (Landau problem) and the same problem in which we added a bidimensional harmonic oscillator potential. Although these problems had already been treated from the point of view of Schwinger's action principle, the novelty of our work relies on the fact that we solved the Heisenberg equations for gauge invariant operators. This procedure has some nice properties, as for instance: (i) the Feynman propagator is obtained in a generic gauge; (ii) the gauge-dependent and gauge-independent parts of the propagator appear clearly separated and (iii) the transformation law for the propagator under gauge transformation can be readly obtained. Besides, we adopted a matrix notation which can be straightforwardly generalized to cases of relativistic charged particles in the presence of constant electromagnetic fields and a plane wave electromagnetic field, treated by Schwinger [2]. For completeness, we showed explicitly how one can obtain the energy spectrum directly from que Feynman propagator. In the Landau problem, we obtained the (infinitely degenerated) Landau levels with the corresponding degeneracy per unit area. For the case where we included the bidimensional harmonic potential, we obtained the energy spectrum after identifying the poles of the corresponding energy Green function. We hope that this pedagogical paper may be useful for undergraduate as well as graduate students and that these two simple examples may enlarge the (up to now) small list of non-relativistic problems that have been treated by such a powerful and elegant method.

\section{Acknowledgments}

F.A. Barone, H. Boschi-Filho and C. Farina would like to thank Professor Marvin Goldberger for a private communication and for kindly sending his lecture notes on quantum mechanics where this method was explicitly used. We would like to thank $\mathrm{CNPq}$ and Fapesp (brazilian agencies) for partial financial support.
[1] F.A. Barone, H. Boschi-Filho, and C. Farina, Am. J. Phys. 71, 483 (2003)

[2] J. Schwinger, Phys. Rev. 82, 664 (1951).

[3] Claude Itzykson and Jean-Bernard Zuber, Quantum Field Theory, (McGraw-Hill Inc., NY, 1980), pg 100.

[4] E.S Fradkin, D.M Gitman, and S.M. Shvartsman, Quantum Eletrodinamics with Unstable Vacuum (Springer, Berlim, 1991).

[5] V. V. Dodonov, I. A. Malkin, and V. I. Manko, Lett. Nuovo Cimento Soc. Ital. Fis. 14, 241 (1975).

[6] V. V. Dodonov, I. A. Malkin, and V. I. Manko, J. Phys. A 9, 1791 (1976).

[7] J. D. Likken, J. Sonnenschein, and N. Weiss, Int. J. Mod. Phys.
A 6, 5155 (1991).

[8] A. Ferrando and V. Vento, Z. Phys. C 63, 485 (1994).

[9] H. Boschi-Filho, C. Farina, and A. N. Vaidya, Phys. Lett. A 215, 109 (1996)

[10] S. P. Gavrilov, D. M. Gitman, and A. E. Goncalves, J. Math. Phys. 39, 3547 (1998).

[11] D. G. C. McKeon, I. Sachs, and I. A. Shovkovy, Phys. Rev. D59, 105010 (1999).

[12] T. K. Chyi, C. W. Hwang, W. F. Kao, G. L. Lin, K. W. Ng, and J. J. Tseng, Phys. Rev. D 62, 105014 (2000).

[13] N. C. Tsamis and R. P. Woodard, Class. Quant. Grav. 18, 83 (2001).

[14] M. Chaichian, W. F. Chen, and R. Gonzalez Felipe, Phys. Lett. 
B 503, 215 (2001)

[15] J. M. Chung and B. K. Chung, Phys. Rev. D 63, 105015 (2001).

[16] H. Boschi-Filho, C. Farina, and A.N. Vaidya, Phys. Let. A 215, 109 (1996).

[17] Luis F. Urrutia and Eduardo Hernandez, Int. J. Theor. Phys. 23, 1105 (1984)

[18] L.F. Urrutia and C. Manterola, Int. J. Theor. Phys. 25, 75 (1986).

[19] N. J. M. Horing, H. L. Cui and G. Fiorenza, Phys. Rev. A 34, 612 (1986)

[20] C. Farina and Antonio Segui-Santonja, Phys. Lett. A 184, 23
(1993).

[21] S.J. Rabello and C. Farina, Phys. Rev. A51, 2614 (1995).

[22] J. Schwinger, Quantum Mechanics: Symbolism of Atomic Measurements, edited by B.G. Englert (Springer, 2001).

[23] Private communication with Professor M. Goldberger. We thank him for kindly sending us a copy of his notes on quantum mechanics given at Princeton for more than ten years.

[24] R.P. Feynman and A.R. Hibbs, Quantum Mechanics and Path Integrals (McGraw-Hill, New York, 1965). 